\title{
Highly efficient direct visible-light-mediated oxidative esterification of aldehydes
}

\author{
Rickard Lindroth ${ }^{1} \cdot$ Hogan P. Bryce-Rogers ${ }^{1} \cdot$ Thomas P. M. Merke $^{1} \cdot$ Carl-Johan Wallentin $^{1}$ (1)
}

Received: 29 October 2021 / Accepted: 14 December 2021 / Published online: 20 January 2022

(c) The Author(s) 2022

\begin{abstract}
A very efficient one-pot procedure for the direct oxidation of aldehydes to esters mediated by visible-light is presented. Utilizing a combination of indium triflate and $\mathrm{N}$-bromosuccinimide, NBS, with either ortho-esters or alcohols provided rapid access to a variety of esters. Certain substrates convert fully within a few seconds, other more challenging tert-butyl esters were formed in good yield after no more than $4 \mathrm{~h}$.
\end{abstract}

\section{Graphical abstract}

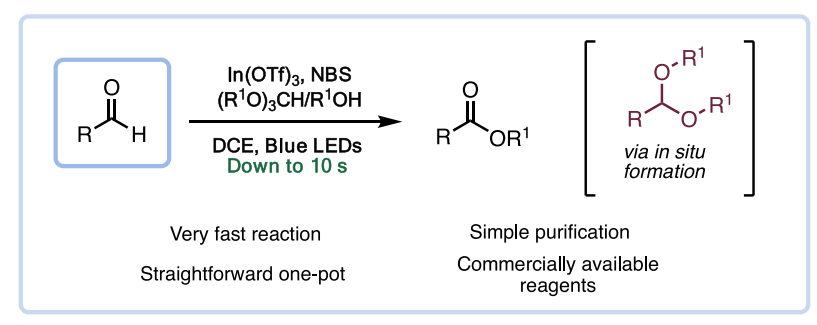

\section{Introduction}

Esters are recognized as indispensable functionalities by virtue of their use in most fields of chemistry. The classical acid-catalyzed Fischer-Speier esterification [1], Scheme 1A, directly converts carboxylic acids to esters with alcohols. Despite its inherent conceptual simplicity, the method is undermined due to the acidic conditions in combination with elevated temperatures typically used, and is thus not applicable in engaging structurally more demanding substrates. Instead, synthetic chemists often turn to various carboxylic acid derivatives or, as in Steglich esterification [2], the addition of a coupling reagent that activates carboxylic acids toward substitution reactions. Common to the aforementioned methods is that the oxidation state + III of the carbonyl carbon already installed a priori. Frequently, however, chemists want to transform groups of lower oxidation states

Carl-Johan Wallentin

carl.wallentin@chem.gu.se

1 Department of Chemistry and Molecular Biology, University of Gothenburg, Kemigården 4, 41296 Gothenburg, Sweden into esters which require either a separate stepwise oxidation of the group to the level necessitated in the foregoing methods or, preferably, the oxidation is conducted in parallel with the esterification.

Several protocols for oxidatively converting aldehydes to esters in one-pot have been reported in literature and their pros and cons thoroughly reviewed by Wolf and coworkers [3]. These methods range from various catalytic approaches, e.g., electro-catalysis, transition metal catalysis and NHC catalysis. Furthermore, various oxidants, in conjunction with a catalyst, have been reported in this context, such as iodine [4, 5] oxone [6], tert-butyl hydroperoxide [7], hydrogen peroxide [8,9], manganese (IV) oxide [10] and TCCA [11]. In more modern approaches $[12,13]$, oxygen from air has been successfully employed in conjunction with NHC catalysis by the Sundén group (Scheme 1B) [14]. Other examples are based on the strategy of transforming the aldehyde in situ to an acetal that subsequently converts oxidatively to an ester $[15,16]$. One such approach was reported by McDonald and co-workers [17] that developed a direct method by converting aldehydes in situ to hemiacetal hypo-iodites using NIS as the 


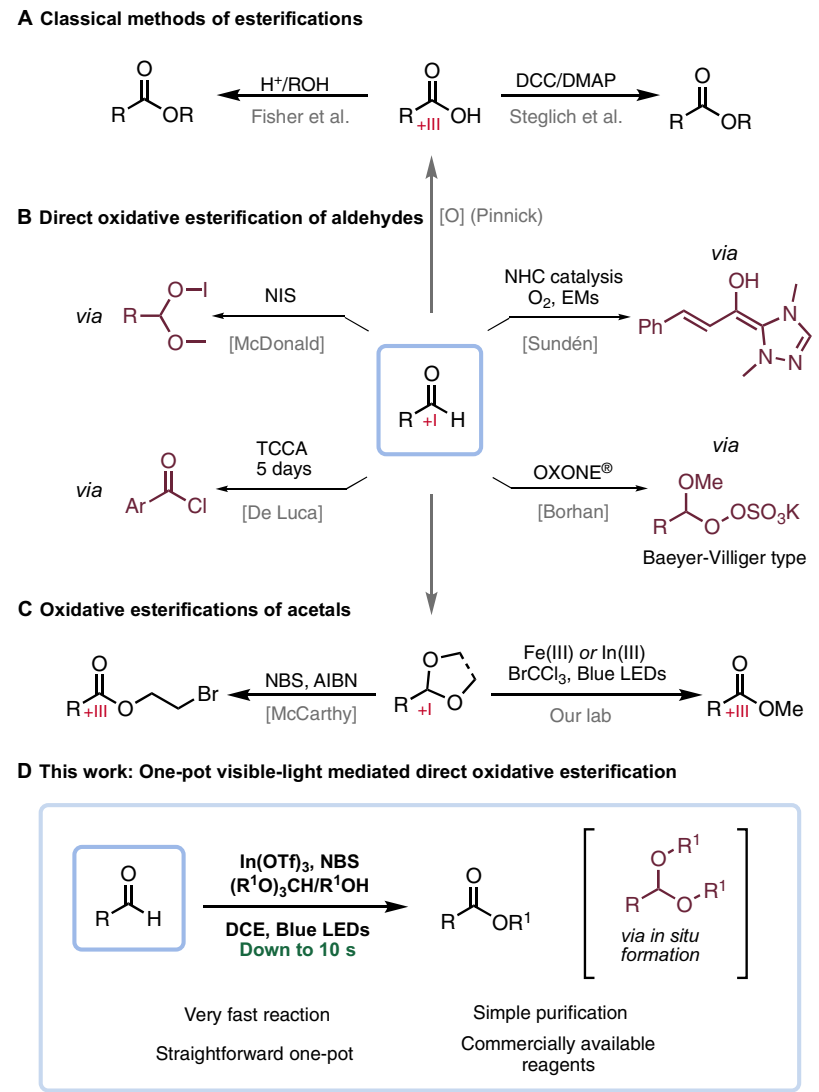

Scheme 1 Various approaches to the formation of esters: A two classical methods, $\mathbf{B}$ oxidative esterification of aldehydes, $\mathbf{C}$ esterification of acetals, D our direct visible-light-mediated esterification of aldehydes

oxidant. Interestingly, this method was not compatible with NCS and only with limited success using NBS. The multitude of methods are complementary in that no single method is without drawbacks, some suffer from expensive or harsh conditions, others from limited substrate scope. Generally, the reaction times are rather long for the majority of the methods ranging from several hours up to 5 days.

As part of our ongoing research efforts, we recently disclosed a visible-light-mediated $\mathrm{Fe}$-catalyzed approach for turning acetals into esters with $\mathrm{BrCCl}_{3}$ serving as the terminal oxidant [18]. Further reports on esterification of acetals exist, utilizing NBS with AIBN initiator [19], UVlight-induced excited-state acetone [20] or peroxides under elevated temperature (Scheme 1C) [21, 22]. Nevertheless, starting directly from acetals can be inconvenient as they rather easily revert to aldehydes on storage and often need to be synthesized. Therefore, we aimed to address this shortcoming by forming acetals in situ and converting these to esters using our visible-light-mediated approach. Furthermore, during our recent efforts, we observed that $\operatorname{In}(\mathrm{OTf})_{3}$, in some cases, also is a competent catalyst for the esterification of acetals. Taken together with the efficient acetalization catalyzed by $\operatorname{In}(\mathrm{OTf})_{3}$ reported by Smith [23], we anticipated that a one-pot visible-light direct oxidative esterification methodology of aldehydes would be feasible (Scheme 1D).

\section{Results and discussion}

For the optimization of this direct esterification, benzaldehyde was chosen as a suitable model substrate. When allowing benzaldehyde to pre-form the acetal by reaction with trimethyl orthoformate (1 eq.) and $\operatorname{In}(\mathrm{OTf})_{3}(1 \mathrm{~mol} \%)$ in DCE for $5 \mathrm{~min}$ followed by addition of $\mathrm{BrCCl}_{3}$ (3 eq.) and irradiation with blue LEDs for a further $18 \mathrm{~h}$ provided methyl benzoate in $32 \%$ yield by ${ }^{1} \mathrm{H}-\mathrm{NMR}$ (see Table 1 ).

A solvent screen showed that DCE provided the methyl benzoate with the highest yield at a concentration of $0.4 \mathrm{M}$ (entry 1).

Next, alternative oxidants other than $\mathrm{BrCCl}_{3}$ were screened and NBS was established as the most competent one providing methyl benzoate in $75 \%$ yield after irradiation with blue LEDs for $18 \mathrm{~h}$ (entry 6). A re-visit of solvents confirmed that DCE is retained as the most suitable solvent also with NBS as the oxidant (entries 7 and 8). Notably, the reaction does not require the use of anhydrous DCE.

Control experiments omitting either the irradiating source (entry 9) or $\operatorname{In}(\mathrm{OTf})_{3}$ (entry 10) showed a significant reduction in yields, $41 \%$ and $13 \%$, respectively. This indicates that both $\mathrm{In}(\mathrm{OTf})_{3}$ and blue light irradiation accelerate the esterification. The latter result is rationalized by a slow, but non-zero, rate of acetal formation in the absence of a Lewis acid. The former result (entry 9) is interesting as it demonstrates that an intermediate acetal is converted to an ester by NBS without the addition of a radical initiator or light, contrary to previously reported procedures [19], and alludes to an $\operatorname{In}(\mathrm{OTf})_{3}$-assisted initiation. In another control experiment, the commercially available benzaldehyde dimethyl acetal was subjected to NBS and blue light irradiation in the absence of $\operatorname{In}(\mathrm{OTf})_{3}$. These conditions still generated the ester in $95 \%$ yield, demonstrating that visible-light itself promotes the reaction with NBS. The same experiment starting from the acetal, but using $\mathrm{BrCCl}_{3}$ as the oxidant, did require $\mathrm{In}(\mathrm{OTf})_{3}$ for the conversion to the ester.

Having established reaction conditions to cleanly transform benzaldehyde to its methyl ester the reaction time was successively reduced down to $1 \mathrm{~h}$ without any loss in efficacy. At this point, the necessity of pre-stirring to form the acetal was reinvestigated, as our initial efforts showed it to be crucial for a clean reaction. Satisfyingly, with the modified conditions, the reaction turned out to work even better simply mixing all reagents together and directly irradiating with blue light to afford the ester in $84 \%$ yield (entry 16 ). 
Table 1 Reaction conditions: benzaldehyde $(0.2 \mathrm{mmol})$, In(OTf $)_{3}(1 \mathrm{~mol} \%)$, brominating agent (1.2-2.0 eq., added after pre-stirring), solvent $(0.5 \mathrm{~mL})$ and trimethyl orthoformate (1.0 eq.) under blue LED irradiation at r.t. for $10 \mathrm{~s}-18 \mathrm{~h}$ under argon atmosphere

\begin{tabular}{|c|c|c|c|c|c|}
\hline \multirow[b]{2}{*}{ Entry } & \multirow[b]{2}{*}{ Brominating agent } & \multicolumn{2}{|c|}{$\begin{array}{c}\left.\ln (\mathrm{OTf})_{3},(\mathrm{MeO})\right)_{3} \mathrm{CH} \\
\text { Oxidant }\end{array}$} & \multirow[b]{2}{*}{$\mathrm{t}_{\text {blue LED }}$} & \multirow[b]{2}{*}{ Yield $^{\mathrm{a}}$ of $1 \mathrm{a} \%$} \\
\hline & & Solvent & $t_{\text {pre-stirring }}$ & & \\
\hline 1 & $\mathrm{BrCCl}_{3}$ & DCE & $5 \mathrm{~min}$ & $18 \mathrm{~h}$ & 32 \\
\hline 2 & $\mathrm{BrCCl}_{3}$ & $\mathrm{DCM}$ & $5 \min$ & $18 \mathrm{~h}$ & 26 \\
\hline 3 & $\mathrm{BrCCl}_{3}$ & $\mathrm{MeCN}$ & $5 \min$ & $18 \mathrm{~h}$ & 14 \\
\hline 4 & $\mathrm{BrCCl}_{3}$ & EtOAc & $5 \mathrm{~min}$ & $18 \mathrm{~h}$ & 3 \\
\hline 5 & Dimethyl 2-bromomalonate & DCE & $5 \mathrm{~min}$ & $18 \mathrm{~h}$ & 33 \\
\hline 6 & NBS & DCE & $5 \min$ & $18 \mathrm{~h}$ & 76 \\
\hline 7 & NBS & DCM & $5 \mathrm{~min}$ & $18 \mathrm{~h}$ & 60 \\
\hline 8 & NBS & $\mathrm{MeCN}$ & $5 \mathrm{~min}$ & $18 \mathrm{~h}$ & 66 \\
\hline 9 & NBS & DCE & $5 \min$ & $18 \mathrm{~h}$ & $41^{\mathrm{b}}$ \\
\hline 10 & NBS & DCE & $5 \mathrm{~min}$ & $18 \mathrm{~h}$ & $13^{\mathrm{c}}$ \\
\hline 11 & NBS & DCE & $5 \mathrm{~min}$ & $8 \mathrm{~h}$ & 74 \\
\hline 12 & NBS & DCE & $5 \min$ & $4 \mathrm{~h}$ & 75 \\
\hline 13 & NBS & DCE & $5 \mathrm{~min}$ & $2 \mathrm{~h}$ & 75 \\
\hline 14 & NBS & DCE & $5 \mathrm{~min}$ & $1 \mathrm{~h}$ & 74 \\
\hline 15 & NBS & DCE & $5 \mathrm{~min}$ & $1 \mathrm{~h}$ & $82^{\mathrm{d}}$ \\
\hline 16 & NBS & DCE & - & $1 \mathrm{~h}$ & 84 \\
\hline 17 & NBS & DCE & - & $5 \mathrm{~min}$ & 91 \\
\hline 18 & NBS & DCE & - & $2 \min$ & 88 \\
\hline 19 & NBS & DCE & - & $10 \mathrm{~s}$ & 82 \\
\hline
\end{tabular}

${ }^{\text {a } Y i e l d ~ o f ~ c o m p o u n d ~} 1$ determined by ${ }^{1} \mathrm{H}$ NMR using dimethyl sulfone or methyl 3,5-dinitrobenzoate as internal standard

${ }^{\mathrm{b}}$ Reaction in the dark

${ }^{\mathrm{c}}$ Reaction without $\mathrm{In}(\mathrm{OTf})_{3}$

${ }^{\mathrm{d}}$ Addition of brominating agent before pre-stirring but no light

Remarkably, reducing the time to 5 min gave the best result with $91 \%$ yield and even at a reaction time of an astonishing $10 \mathrm{~s}$, methyl benzoate was formed in excellent yield of $82 \%$ (entries 17 and 19, respectively). Thus, the optimized reaction for the photo-mediated direct esterification of aldehydes involved the conditions: trimethyl orthoformate (1.0 eq.), $\mathrm{In}(\mathrm{OTf})_{3}(1 \mathrm{~mol} \%)$, NBS (1.2 eq.), DCE $(0.5 \mathrm{~mL})$ and blue LEDs irradiation under stirring for $5 \mathrm{~min}$ (91\%, entry 19$)$.

With the optimized reaction conditions at hand, we next turned our attention to the scope of the aromatic moiety of the aldehydes (Scheme 2). First, methyl benzoate (1a) could be isolated in $71 \%$ yield after irradiating the reaction for $10 \mathrm{~s}$, and yet a better yield of $91 \%$ could be achieved when employing the standard conditions of $5 \mathrm{~min}$. Care should be taken as methyl benzoate seemed abnormally volatile, possibly due to a formed azeotropic mixture.

The reaction demonstrates a broad scope in terms of electronic effects with all isolated yields ranging between 71 and 99\%. Electron-rich 4-methoxy benzaldehyde was converted to (1b) and isolated in $91 \%$ yield and its congener 4-trifluoromethoxy benzaldehyde in a good $81 \%$ yield (1f). Electron-deficient 4 -formylbenzonitrile smoothly converted to the ester (1d) in also $81 \%$ yield. For the more strongly electron-deficient 4-nitro benzaldehyde and 2-nitro benzaldehyde, the reaction time needed extension to $1 \mathrm{~h}$, although, with excellent yields of $91 \%$ and $99 \%$, respectively. The longer reaction time for these substrates is likely due to a slower dissociation to form an intermediate cation (see Scheme 5), greatly destabilized by such potent electron withdrawing groups. When subjecting 2-formylbenzoic acid to the reactions, both the aldehyde and carboxylic acid functionalities transformed into methyl esters (1j) wherefore two equivalents of trimethyl orthoformate were crucial for obtaining a clean reaction affording the di-ester in $80 \%$ yield. The reaction also proceeds well with fused ring systems and heteroaromatics as exemplified by (1k) and (11).

Next, we aimed for conditions that could diversify the ester functionalities beyond methyl esters (Scheme 3). Substituting the trimethyl orthoformate for triethyl orthoformate 


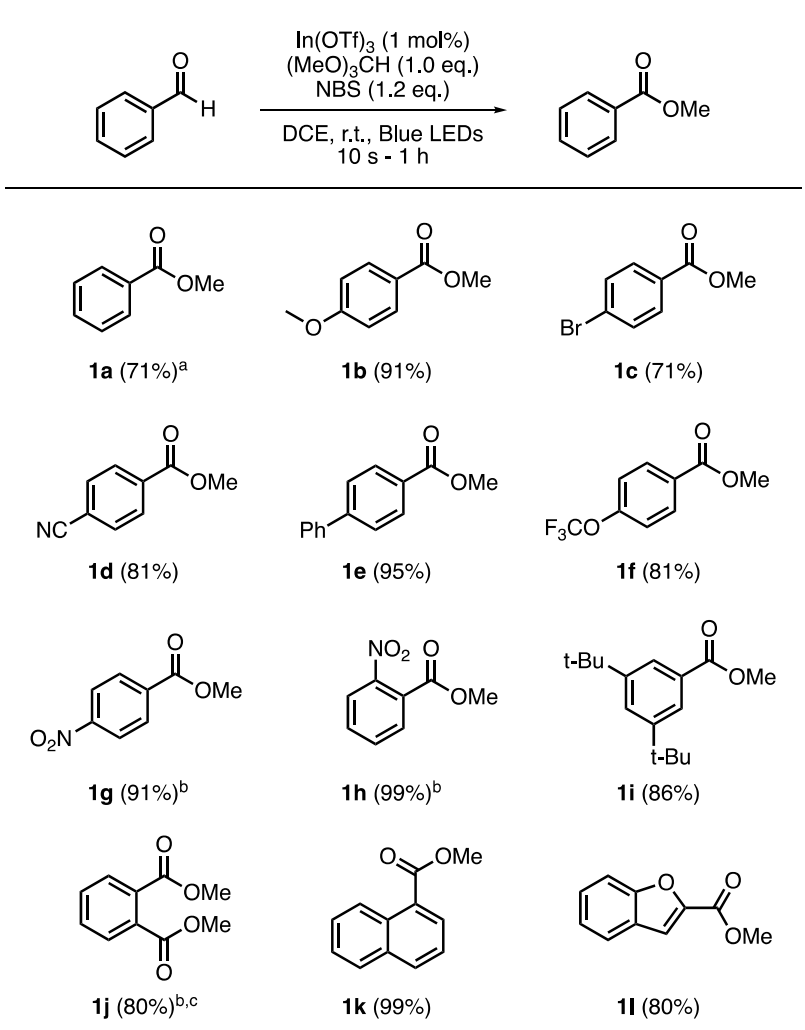

Scheme 2 Reactions were conducted at the scale of $0.2 \mathrm{mmol}$ with respect to the aldehyde. $0.4 \mathrm{M}$ concentration and irradiation with blue LEDs for $5 \mathrm{~min}$, unless otherwise specified. Yields are those of isolated products. ${ }^{\mathrm{a}}$ Reaction time $10 \mathrm{~s} .{ }^{\mathrm{b}}$ Reaction time $1 \mathrm{~h} .{ }^{\mathrm{c}} 2$ eq. NBS

afforded the corresponding ethyl ester in $89 \%$ yield (2a). There is a greater variety of alcohols than ortho-esters which would enable a more extensive product scope if conditions also allowed for the direct use of alcohols. When primary alcohol isobutyl alcohol (2 eq.) is used the corresponding isobutyl ester (2a) was formed in $95 \%$ yield. Remarkably, using tert-butanol gave the tert-butyl ester in very good yield $(81 \%, \mathbf{2 c})$. To succeed with this reaction utilizing alcohols, the reaction time needed to be extended to $4 \mathrm{~h}$, and the stoichiometry of NBS to two, which still provides satisfying conditions for this challenging tert-butyl ester synthesis.

In situ formation of cyclic acetals would result in a doubly functionalized ester by the additional incorporation of a bromide, suitable for further functionalization. When ethylene glycol was used the 2-bromoethyl 4-biphenyl carboxylate (2d) was obtained in a moderate $59 \%$ yield. Additionally, 1,3-propanediol and 1,2-cyclohexanediol were evaluated, yielding the corresponding products in $37 \%$ and $22 \%$ yields, respectively. However, the significant drops in yields are attributed to the isolatable coformation of di-ester dimers, formed in ratio 95:5, 80:20, and 71:29, respective from $\mathbf{2 d}, \mathbf{2 e}$ and $\mathbf{2 f}$.

Next, we investigated the opportunity of engaging aliphatic aldehydes in the esterification. Aldehydes with a

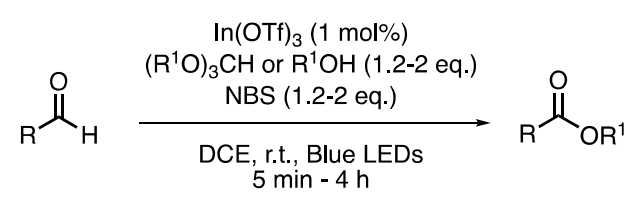

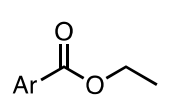

$2 a(89 \%)^{a, d}$<smiles>O=C([Al])OCCBr</smiles>

$2 d(59 \%)^{a, d}$

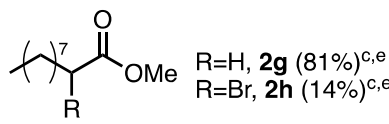<smiles>COC(=O)C1(P)CCCCC1</smiles><smiles>[14CH3]C(=O)OCCCBr</smiles>

$2 e(37 \%)^{c, d}$<smiles>CC(C)(C)OC(=O)[Te]</smiles>

$2 c(81 \%)^{b, e}$<smiles>O=C([Al])OC1CCCCC1Br</smiles>

2f $(22 \%)^{c, d}$<smiles>CC(C)(C)C(=O)OC(OC(=O)C(C)(C)C)C(C)(C)C</smiles>

2k $(51 \%)^{c, e}$
Scheme 3 Reactions were conducted at $0.2 \mathrm{mmol}$ scale, $0.4 \mathrm{M}$ concentration and irradiated with blue LEDs. Yields are those of isolated products. $\mathrm{Ar}=4$-biphenyl-. ${ }^{\mathrm{a}}$ Reaction time $=5 \mathrm{~min},{ }^{\mathrm{b}}$ Reaction time $=4 \mathrm{~h},{ }^{\mathrm{c}}$ Reaction time $=1 \mathrm{~h} .{ }^{\mathrm{d}} 1.2$ eq. NBS, ${ }^{\mathrm{e}} 2$ eq. NBS

secondary or tertiary $\alpha$-carbon underwent esterification within $1 \mathrm{~h}$ reaction time. However, these substrates also produced $\alpha$-brominated esters as the main product for tertiary substrates ( $\mathbf{2} \mathbf{i}$ vs $\mathbf{2} \mathbf{j})$ and as a minor product for secondary substrates ( $\mathbf{2}$ vs $\mathbf{2 h}$ ). It was necessary to use

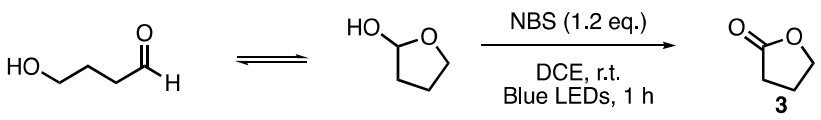

Scheme 4 Lactone formation by oxidation of a hemiacetal

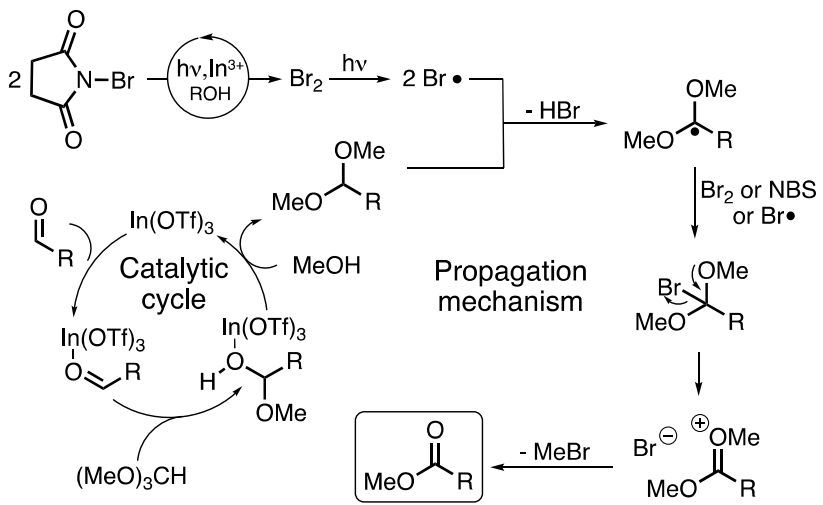

Scheme 5 Proposed mechanism 
2 eq. of NBS for full conversion of the aldehydes and bromination could not be prevented by decreasing the equivalents of NBS as the bromination occurred in competition with the esterification process. For quaternary $\alpha$-carbons, an unexpected compound of proposed structure $2 \mathbf{k}$ was formed as the sole product in $51 \%$ yield.

With the investigation into aliphatic aldehydes came the interest of application toward cyclized hemiacetals. Indeed, the cyclic hemiacetal (Scheme 4) converted to the lactone 3 ( $80 \%$ yield); however, the formation was observed also in the absence of $\operatorname{In}(\mathrm{OTf})_{3}$. Consequently, in the case where an acetal or hemiacetal can form readily, there is no necessity for a Lewis acid to promote the esterification process.

A plausible mechanism is depicted in Scheme 5. The first step of the mechanism is a proposed Lewis acid-catalyzed acetalization of the aldehyde by $\operatorname{In}(\mathrm{OTf})_{3}$. However, we cannot rule out the possibility of the reaction proceeding from an intermediary hemiacetal. Next, visible-lightinduced homolytic cleavage of molecular bromine formed from NBS affords two bromine radicals that can abstract the activated hydrogen from the acetal (or hemiacetal) from where a propagating mechanism follows. UV-Vis experiments revealed a very efficient formation of molecular bromine from NBS in the presence of alcohol under blue light irradiation. Even trace amounts of alcohol, present when utilizing orthoformate, are enough to efficiently mediate this reaction. In addition, $\operatorname{In}(\mathrm{OTf})_{3}$ further accelerates this process (see SI for experimental details). The so-formed acetal radical abstracts a bromine from NBS (or molecular bromine) to form an unstable intermediate that collapses by expelling a bromide ion and, thus, forms the carbonyl group. Finally, the so-formed bromide ion acts as a nucleophile and in a substitution reaction demethylates the carbonyl oxygen to give the desired ester.

In summary, a simple and highly efficient visible-lightmediated direct esterification of aldehydes, via acetals or hemiacetals, was developed using $\operatorname{In}(\mathrm{OTf})_{3}$ as a catalyst in conjunction with NBS as oxidant (and brominating agent for intermediate cyclic acetals). For some substrates, the reaction goes practically to completion within a few seconds. Other substrates still converted rapidly to esters but required $5 \mathrm{~min}$ to $1 \mathrm{~h}$ of stirring under irradiation. The reaction shows an extensive scope of different electronic properties of various benzaldehydes. Furthermore, the reaction can employ various alcohols, including bulky tert-butanol, to form the corresponding esters. Some limitations in selectivity were observed in the case for aliphatic aldehydes that, along with the ester, also produced $\alpha$-brominated esters to various extents. Such limitation could conceivably be addressed by looking at alternative oxidants suitable within the context of visible-light and $\operatorname{In}(\mathrm{OTf})_{3}$ catalysis.
Supplementary Information The online version contains supplementary material available at https://doi.org/10.1007/s43630-021-00160-5.

Acknowledgements The Swedish Research Council is acknowledged for financial support. Dr. Andrew Carrod is acknowledged for recording emission spectra of our lamps and assistance with UV-Vis experiments.

Funding Open access funding provided by University of Gothenburg.

\section{Declarations}

Conflict of interest The authors declare no interest of conflict.

Open Access This article is licensed under a Creative Commons Attribution 4.0 International License, which permits use, sharing, adaptation, distribution and reproduction in any medium or format, as long as you give appropriate credit to the original author(s) and the source, provide a link to the Creative Commons licence, and indicate if changes were made. The images or other third party material in this article are included in the article's Creative Commons licence, unless indicated otherwise in a credit line to the material. If material is not included in the article's Creative Commons licence and your intended use is not permitted by statutory regulation or exceeds the permitted use, you will need to obtain permission directly from the copyright holder. To view a copy of this licence, visit http://creativecommons.org/licenses/by/4.0/.

\section{References}

1. Fischer, E., \& Speier, A. (1895). Darstellung der Ester. Berichte der Deutschen Chemischen Gesellschaft, 28(3), 3252-3258.

2. Neises, B., \& Steglich, W. (1978). Simple method for the esterification of carboxylic acids. Angewandte Chemie International Edition English, 17(7), 522-524.

3. Ekoue-Kovi, K., \& Wolf, C. (2008). One-pot oxidative esterification and amidation of aldehydes. Chemistry A European Journal, 14(21), 6302-6315.

4. Mori, N., \& Togo, H. (2005). Facile oxidative conversion of alcohols to esters using molecular iodine. Tetrahedron, 61(24), $5915-5925$.

5. Yamada, S., Morizono, D., \& Yamamoto, K. (1992). Mild oxidation of aldehydes to the corresponding carboxylic acids and esters: Alkaline iodine oxidation revisited. Tetrahedron Letters, 33(30), 4329-4332.

6. Travis, B. R., Sivakumar, M., Hollist, G. O., \& Borhan, B. (2003). Facile oxidation of aldehydes to acids and esters with oxone. Organic Letters, 5(7), 1031-1034.

7. Zhu, Y., Yan, H., Lu, L., Liu, D., Rong, G., \& Mao, J. (2013). Copper-catalyzed methyl esterification reactions via $\mathrm{C}-\mathrm{C}$ bond cleavage. Journal of Organic Chemistry, 78(19), 9898-9905.

8. Talukdar, D., Sharma, K., Bharadwaj, S. K., \& Thakur, A. J. (2013). $\mathrm{VO}(\mathrm{acac})_{2}$ : An efficient catalyst for the oxidation of aldehydes to the corresponding acids in the presence of aqueous $\mathrm{H}_{2} \mathrm{O}_{2}$. Synlett, 24(8), 963-966.

9. Espenson, J., Zhu, Z., \& Zauche, T. (1999). Bromide ions and methyltrioxorhenium as cocatalysts for hydrogen peroxide oxidations and brominations. Journal of Organic Chemistry, 64(4), 1191-1196.

10. Maki, B. E., \& Scheldt, K. A. (2008). N-heterocyclic carbenecatalyzed oxidation of unactivated aldehydes to esters. Organic Letters, 10(19), 4331-4334. 
11. Gaspa, S., Porcheddu, A., \& De Luca, L. (2015). Metal-free direct oxidation of aldehydes to esters using TCCA. Organic Letters, 17(15), 3666-3669.

12. Yu, C., Özkaya, B., \& Patureau, F. (2021). Electro-oxidative selective esterification of methylarenes and benzaldehydes. Chemistry A European Journal, 27(11), 3682-3687.

13. Wojcieszak, R., et al. (2020). Aerobic oxidation of 1,6-hexanediol to adipic acid over Au-based catalysts: The role of basic supports. Catalysts, 10(4), 1-14.

14. Ta, L., Axelsson, A., \& Sundén, H. (2016). Attractive aerobic access to the a, b-unsaturated acyl azolium intermediate: Oxidative NHC catalysis via multistep electron transfer. Green Chemistry, 18(3), 686-690.

15. Rhee, H., \& Kim, J. Y. (1998). A simple one-pot procedure for the conversion of aldehydes to methyl esters. Tetrahedron Letters, 39, $1365-1368$.

16. Aoyama, T., Takido, T., \& Kodomari, M. (2005). One-pot synthesis of w-bromoesters from aromatic aldehydes and diols using pyridinium hydrobromide perbromide. Tetrahedron Letters, 46(12), 1989-1992.
17. McDonald, C., Holcomb, H., Kennedy, K., Kirkpatrick, E., Leathers, T., \& Vanemon, P. (1989). $N$-Iodosuccinimide-mediated conversion of aldehydes to methyl esters. Journal of Organic Chemistry, 54(5), 1213-1215.

18. Submitted manuscript.

19. Prugh, J. D., \& McCarthy, W. C. (1966). The oxidation of acetals with $n$-bromosuccinimide. Tetrahedron Letters, 7(13), 1351-1356.

20. Elad, D., \& Youssefyeh, R. (1963). The photochemical conversion of acetals to carboxylic esters. Tetrahedron Letters, 4(30), 2189-2191.

21. Matsumoto, M., Kobayashi, H., \& Hotta, Y. (1984). Acid-catalyzed oxidation of benzaldehydes to phenols by hydrogen peroxide. Journal of Organic Chemistry, 49, 4741-4743.

22. Huyser, E. S., \& Garcia, Z. (1962). Peroxide-induced conversions of cyclic acetals of benzaldehyde to benzoate esters. Journal of Organic Chemistry, 27(8), 2716-2719.

23. Smith, B. M., \& Graham, A. E. (2006). Indium triflate mediated acetalization of aldehydes and ketones. Tetrahedron Letters, 47(52), 9317-9319. 